\title{
Circulating S100A4 as a prognostic biomarker for patients with nonparoxysmal atrial fibrillation after catheter ablation
}

\author{
Lijun Qian $^{1 \#}$, Jinlong Gong ${ }^{2 \#}$, Wenjie $\mathrm{Ma}^{1}$, Yan Sun ${ }^{1}$, Jian Hong ${ }^{1}, \mathrm{Di}_{\mathrm{Xu}}{ }^{1}, \mathrm{Ming} \mathrm{Chu}^{3}$ \\ ${ }^{1}$ Department of Geriatrics, The First Affiliated Hospital of Nanjing Medical University, Nanjing, China; ${ }^{2}$ Department of Cardiology, Jiangdu \\ People's Hospital Affiliated to Medical College of Yangzhou University, Yangzhou, China; ${ }^{3}$ Department of Cardiology, The First Affiliated Hospital \\ of Nanjing Medical University, Nanjing, China \\ Contributions: (I) Conception and design: L Qian, J Gong, M Chu; (II) Administrative support: M Chu, D Xu; (III) Provision of study materials or \\ patients: L Qian, W Ma, Y Sun, J Hong; (IV) Collection and assembly of data: L Qian, J Gong, M Chu; (V) Data analysis and interpretation: L Qian, \\ J Gong, M Chu; (VI) Manuscript writing: All authors; (VII) Final approval of manuscript: All authors. \\ \#These authors contributed equally to this work. \\ Correspondence to: Ming Chu, MD. Department of Cardiology, The First Affiliated Hospital of Nanjing Medical University, No. 300 Guangzhou \\ Road, Nanjing 210029, China. Email: chuming@njmu.edu.cn.
}

Background: Atrial fibrosis is involved in non-paroxysmal atrial fibrillation (NPAF) and is mainly mediated by the calcium-binding protein S100A4. This study aimed to verify the role of circulating S100A4 in the diagnosis of atrial fibrosis and the prognosis of NPAF.

Methods: Consecutive NPAF patients undergoing catheter ablation were selected. Patients with low voltage amplitudes $(<0.40 \mathrm{mV})$ in the left atrium (LA), defined as low voltage zones (LVZs), were grouped in the scar group by electroanatomic mapping (EAM). Circulating S100A4 was detected by a human enzyme-linked immunosorbent assay (ELISA). The role of S100A4 in atrial fibrosis was further evaluated by Masson's trichrome staining and immunochemistry (IHC) in NPAF (atrial pacing) and control dogs. The prognostic value of the circulating S100A4 was evaluated by Cox regression analyses, the Kaplan-Meier (KM) method, and receiver operating characteristic (ROC) curves.

Results: We enrolled a total of $101 \mathrm{NPAF}$ patients (age $60 \pm 8$ years) who underwent EAM, including 53 patients with scars and 48 patients without scars at 1-year follow-up. The scar group showed a higher serum level of S100A4 $(3.4 \pm 1.7$ vs. $2.5 \pm 1.4 \mathrm{ng} / \mathrm{mL}, \mathrm{P}<0.001)$ than the non-scar group. In the canine model, scar size matched the larger location of interstitial fibrosis in the NPAF group determined by Masson's trichrome staining. The expression of $\alpha$-SMA and S100A4 was elevated in the NPAF group as determined by IHC compared to the control group $(\mathrm{P}<0.001)$. The clinical recurrence rate was markedly elevated in the scar group $(27.1 \%$ vs. $8.9 \%, \mathrm{P}<0.001)$, and the area under the ROC curve was high $(0.865,95 \% \mathrm{CI}$ : $0.750-0.981$ ) in predicting clinical recurrence of NPAF with the circulating S100A4 model.

Conclusions: Circulating S100A4 plays a role in atrial fibrosis in NPAF patients following ablation. The level of serum S100A4 can predict the clinical recurrence of NPAF.

Keywords: Atrial fibrillation (AF); catheter ablation; electroanatomic mapping (EAM); S100A4; atrial fibrosis

Submitted Mar 09, 2021. Accepted for publication Jul 14, 2021.

doi: 10.21037/atm-21-1101

View this article at: https://dx.doi.org/10.21037/atm-21-1101

^ ORCID: 0000-0002-8773-2065. 


\section{Introduction}

Atrial fibrillation (AF), the most prevalent clinical tachyarrhythmia, leads to high morbidity and mortality (1). Atrial fibrosis is a hallmark of persistent non-paroxysmal atrial fibrillation (NPAF) (2), and NPAF patients show a 2-to-3-fold elevation in fibrosis levels $(3,4)$. However, it remains unclear how atrial fibrosis drives NPAF in the adult left atrium (LA).

Electroanatomic mapping (EAM) is a new method for detecting atrial fibrosis in patients with NPAF. By preventing myocardial cell coupling and reducing conduction velocity, atrial fibrosis produces lower amplitude electrograms, electrogram fractionation, and conduction heterogeneity (5). Thus, fibrotic changes are translated into abnormal electrical signals that can be targeted and measured by three-dimensional EAM during sinus rhythm (SR) (6). More importantly, the low voltage points coalesce, becoming contiguous to form a low voltage zone (LVZ) $(7,8)$. As previously reported, the LVZ was defined as the LA scar (9). However, it remains unclear whether it has a prognostic value in atrial fibrosis.

S100 calcium-binding protein A4 (S100A4) is a member of the $\mathrm{S} 100$ superfamily of EF-hand calcium-binding proteins (10). It is deregulated in fibrosis of the lung (11), liver (12), kidney (13), and heart (14) and participates in cellular processes, especially cell differentiation and growth. Our preliminary study revealed that down-regulation of S100A4 alleviated cardiac fibrosis in a myocardial infarction (MI) murine model (15), and Kato et al. found cardiac fibroblast accumulation through an endothelial-mesenchymal transition in the atrium of patients with AF (16). However, the underlying mechanisms have not been elucidated whether circulating S100A4 is elevated in atrial fibrosis in NPAF patients.

This study applied EAM to identify LA scars in NPAF patients who underwent catheter ablation at 1 -year followup. We conducted a case-control study to determine the relationship between circulating S100A4 and atrial fibrosis in NPAF patients and verified the findings with an NPAF canine model. Then, the prognostic role of circulating S100A4 in clinical NPAF recurrence was evaluated. We present the study following the STARD reporting checklist (available at https://dx.doi.org/10.21037/atm-21-1101).

\section{Methods}

\section{Patients and ethics}

The study was conducted in accordance with the Declaration of Helsinki (as revised in 2013). The study was approved by the Ethics Committee of the First Affiliated Hospital of Nanjing Medical University (2018-SR-229). All procedures complied with the institutional guidelines of the First Affiliated Hospital of Nanjing Medical University Health System, and all patients signed written consent forms. This study was designed as a retrospective study. A total of 101 consecutive NPAF patients who underwent catheter ablation between January 2017 and August 2018 were enrolled. The inclusion criteria were as follows: (I) patients who were clinically diagnosed with NPAF and underwent catheter ablation in our hospital, and (II) EAM was performed during the operation. The exclusion criteria were those with tumors, diabetes [which may contribute to cardiac fibrosis and was an interference factor in patients with NPAF (17)], inflammatory diseases, infectious diseases, fibrotic diseases in vital organs (lung, kidney and liver), or a history of MI.

\section{Ablation protocol}

Entrance and exit blocks were used as the electrophysiological endpoints to isolate ipsilateral circumferential pulmonary veins (PVs). Cavotricuspid isthmus (CTI) ablation was then performed in all patients. Subsequently, chemical cardioversion was performed to restore SR. As previously described, if cardioversion was unsuccessful, ablation was continued using the STEPWISE approach (18). Linear ablation at the LA roof, the posterior mitral leaflet, coronary sinus roof, and CTI followed by ablation of complexfractionated electrograms were sequentially performed as deemed necessary by the operator to achieve termination of AF. PV isolation and a bidirectional block across all linear lesions were confirmed during SR at the end of the procedure. Otherwise, activation and entrainment mapping were performed to identify the optimal site for ablation. In the study, all enrolled patients underwent PV isolation and CTI ablation.

\section{EAM}

Once SR was restored, EAM was performed using the EnsiteNavX System (St. Jude Medical) $(5,18)$. Bipolar signals between the distal electrode pair were recorded (filtered at 30 to $400 \mathrm{~Hz}$ ) and displayed at $100 \mathrm{~mm} / \mathrm{s}$. Subsequently, peak-to-peak amplitude of the bipolar electrogram was measured automatically in the system. Of note, $95 \%$ of all bipolar electrogram signals recorded from the normal LA were $>0.38 \mathrm{mV}$. Using the data generated 

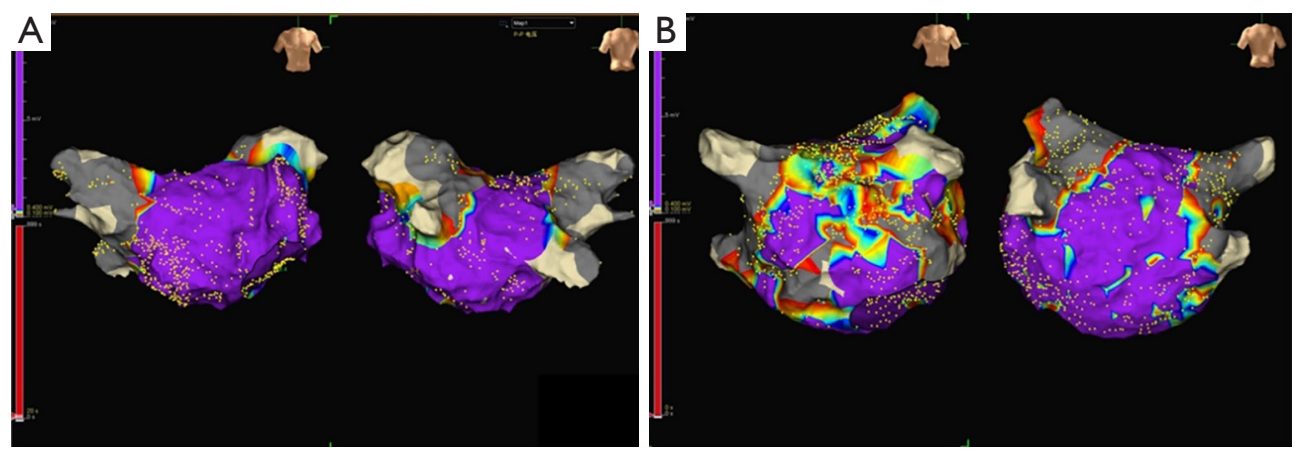

Figure 1 LA voltage mapping in sinus rhythm following pulmonary vein antrum isolation in chest PA and AP views. Purple represents bipolar peak-to-peak electrogram voltage $>0.40 \mathrm{mV}$. In (A), minimal or no LVZ was presented whereas in (B), there were LVZs affecting the LA anterior wall. LA, left atrial; PA, posteroanterior; AP, anteroposterior; LVZ, low voltage zone.

from the control group, we defined normal endocardium using NavX contact mapping as a bipolar electrogram of $>0.4 \mathrm{mV}$ in our previous study. The point with voltage $\leq 0.4 \mathrm{mV}$ was arbitrarily defined as the low voltage point. If the low voltage points coalesced and were contiguous to form a zone, we analyzed the distribution of the $\operatorname{LVZ}(7,8)$. The scar group was defined as patients with an LVZ in the LA through EAM. Finally, a total of 101 patients were assigned to the non-scar $(\mathrm{n}=48$, Figure $1 A)$ and NPAF scar (with LVZs, n=53, Figure 1B) groups.

\section{Laboratory procedures}

All tests were carried out in triplicate and repeated at least twice. A 6-mL blood sample from each subject was collected at baseline (within 24 hours before catheter ablation) for the study. Then, samples were centrifuged at 3,000 r/min for 15 minutes, coded, and stored at $-70{ }^{\circ} \mathrm{C}$ until analysis. Serum total cholesterol (TC), total triglyceride (TG), highdensity lipoprotein (HDL), low-density lipoprotein (LDL), fasting blood sugar (FBS), and brain natriuretic peptide (BNP) were measured with enzymatic methods. Circulating S100A4 was measured by an enzyme-linked immunosorbent assay (ELISA) kit (SEC020Hu, Cloud-Clone Crop, CST, USA). After incubation, absorbance was measured at a wavelength of $450 \mathrm{~nm}$.

\section{Clinical follow-up}

All patients were required to make clinic visits and 24-hour Holter recordings at 1, 3, 6, and 12 months post-ablation in the first year and every 6 months in subsequent years. Oral anticoagulation therapy was maintained in all patients for at least 3 months after ablation. After that, the CHA2DS2VASc score was used to assess the efficacy of anticoagulation therapy. Antiarrhythmic drugs were discontinued 3 months after the anticoagulation indexes had normalized. This was followed by selecting the features, including the histogram features, geometry features, and texture features. All clinical information and reference standard results were not available to neither the performers/readers of the index test nor the assessors of the reference standard.

\section{Canine surgery}

Six adult dogs (weight $12.2 \pm 1.6 \mathrm{~kg}$ ) of either sex were randomly assigned to the NPAF and control group ( $n=3$ per group). The animal study was approved by the ethical committee (approval No. YFYDW2017004) and carried out according to relevant regulations of the First Affiliated Hospital of Nanjing Medical University. All animal experiments were performed following the guidelines published by National Institutes of Health (No. 85-23, revised 1996).

Briefly, dogs were anesthetized with intravenous pentobarbital (25 mg/ $\mathrm{kg}$ ) and additional doses of $2 \mathrm{mg} / \mathrm{kg}$ per hour postinjection. A spiral pacemaker electrode lead (SetroxS60, Biotronik, Germany) was placed through the right femoral vein in the right appendage, where the pacing threshold was less than $1.0 \mathrm{~V}$, and pacemaker impedance was less than $800 \Omega$. The ends of the electrode lead were tunneled subcutaneously and exposed to the right groin subcutaneous pouch. To induce continuous rapid atrial pacing (400 bpm) for 4 weeks after 1 week of normal rehabilitation feeding, a high-frequency pacemaker (AF model pacemakers, Lepu Medical Electronic Technology Co., Ltd.) was implanted in the right groin subcutaneous pouch and fixed. 


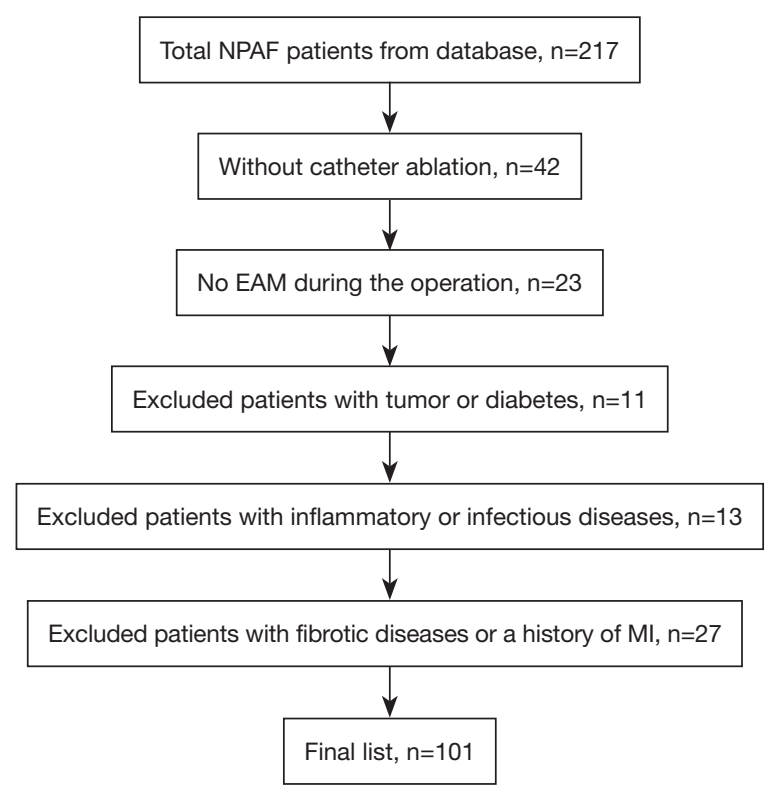

Figure 2 Patient inclusion and exclusion diagram. The algorithm of patients included in the study after excluding patients not meeting the inclusion criteria. See text for details with regard to the exclusion criteria. NPAF, non-paroxysmal atrial fibrillation; EAM, electroanatomic mapping; MI, myocardial infarction.

\section{Masson's trichrome staining}

The atrial tissues were fixed in $4 \%$ buffered formaldehyde, embedded in paraffin, and then prepared into 5 - $\mu \mathrm{m}$-thick sections. Subsequently, we performed Masson's trichrome staining to investigate the distribution and the extent of the fibrosis.

\section{Immunobistochemistry}

The atrial tissues were prepared into 5 - $\mu$ m-thick paraffin sections, then deparaffinization and antigen retrieval were performed in a steam cooker in $1 \mathrm{mM}$ EDTA ( $\mathrm{pH}$ 9.0) for 15 minutes. Slides were then treated with $5 \%$ bovine serum albumin (BSA) at room temperature for 1 hour, the appropriate primary antibodies at $4{ }^{\circ} \mathrm{C}$ overnight, and then universal secondary antibodies at room temperature for 30 minutes. Chromogens and hematoxylin were used for counterstaining before mounting.

\section{Statistical analysis}

All data were analyzed with SPSS 24.0 (Chicago, USA) and GraphPad Prism 6.0 software (CA, USA). Considering type I error $=0.05$, study power $=0.8$, assuming the relative risk of atrial scar $=3$, and at least a $20 \%$ dropout rate, the study sample size was calculated as 20 subjects per group. Sampling calculations were performed until the least calculated sample size in each group was achieved. Therefore, the sample size of our current study met the criteria for statistical power for analysis.

Values were expressed as mean \pm standard deviation, and $\mathrm{P}<0.05$ was considered statistically significant. Continuous variables were compared using the Student's $t$-test or oneway ANOVA test. A Cox proportional hazard model was utilized to compare hazard ratios of events between groups. Kaplan-Meier curves were plotted to depict event-free survival. Receiver operating characteristic (ROC) curves calculated the specificity and sensitivity across the range of possible cutoff values of risk scores were calculated by ROC curves. The area under the curve (AUC) and 95\% confidence interval (CI) were calculated to analyze the overall discriminative ability of risk scores.

\section{Results}

\section{Baseline characteristics}

A total of 227 patients were scanned initially, while 42 cases without ablation, 23 cases without EAM tests, 11 cases with tumors or diabetes, 13 cases with inflammatory or infectious diseases, and 27 patients with fibrotic diseases or a history of MI were excluded. As shown in Figure 2, 101 consecutive NPAF patients (ages $60 \pm 8$ years, follow-up $12 \pm 6$ months) were recruited, including 53 patients with scars and 48 patients without scars at 1-year follow-up. The baseline characteristics of the entire cohort are shown in Table 1. We found no significant differences in clinical characteristics, including body mass index (BMI), systolic blood pressure (SBP), diastolic blood pressure (DBP), TC, TG, HDL, LDL, and FBS levels between the scar and non-scar group (all $\mathrm{P}>0.05$ ).

\section{S100A4 activated atrial fibrosis in NPAF patients}

Serum S100A4 levels were detected by ELISA analysis. Figure $3 A$ showed that the serum level of S100A4 was significantly higher in the NPAF scar group $(3.4 \pm 1.7$ vs. $2.5 \pm 1.4 \mathrm{ng} / \mathrm{mL}$, $\mathrm{P}<0.001)$ than in the non-scar group, and the cutoff value of the serum S100A4 level was calculated as $2.9 \mathrm{ng} / \mathrm{mL}$. This suggested the significant elevation of circulating S100A4 in NPAF patients with atrial fibrosis. In the canine model, scar size matched the larger location of interstitial fibrosis in the 
Table 1 Baseline indexes of the scar and non-scar groups

\begin{tabular}{|c|c|c|c|c|}
\hline Indexes & All patients $(n=101)$ & Non-scar $(n=48)$ & Scar $(n=53)$ & $P$ value \\
\hline Male, n [\%] & $78[77]$ & $43[90]$ & $35[66]$ & 0.13 \\
\hline Duration of AF persistence (month) & $17 \pm 16$ & $19 \pm 17$ & $15 \pm 14$ & 0.21 \\
\hline Alcohol intake, n [\%] & 69 [68] & 29 [60] & 39 [74] & 0.08 \\
\hline Hypertension, n [\%] & $41[41]$ & $19[40]$ & $22[42]$ & 0.27 \\
\hline Coronary heart disease, $\mathrm{n}[\%]$ & $10[10]$ & $4[8]$ & $6[11]$ & 0.19 \\
\hline $\mathrm{BMI}\left(\mathrm{kg} / \mathrm{m}^{2}\right)$ & $24 \pm 3$ & $24 \pm 2$ & $24 \pm 3$ & 0.64 \\
\hline $\mathrm{SBP}(\mathrm{mmHg})$ & $124 \pm 10$ & $125 \pm 10$ & $123 \pm 9$ & 0.50 \\
\hline $\mathrm{TG}(\mathrm{mg} / \mathrm{dL})$ & $130 \pm 13$ & $130 \pm 14$ & $132 \pm 11$ & 0.20 \\
\hline $\mathrm{HDL}(\mathrm{mg} / \mathrm{dL})$ & $39 \pm 7$ & $39 \pm 7$ & $38 \pm 7$ & 0.59 \\
\hline LDL (mg/dL) & $118 \pm 8$ & $119 \pm 8$ & $117 \pm 9$ & 0.23 \\
\hline FBS (mg/dL) & $118 \pm 19$ & $119 \pm 19$ & $119 \pm 18$ & 0.89 \\
\hline
\end{tabular}

Mean \pm SD was reported. AF, atrial fibrillation; BMI, body mass index; SBP, systolic blood pressure; DBP, diastolic blood pressure; TC, total cholesterol; TG, total triglyceride; HDL, high-density lipoprotein; LDL, low-density lipoprotein; FBS, fasting blood sugar.

NPAF group by Masson's trichrome staining (Figure 3B). The expression of $\alpha$-SMA (Figure 3C) and S100A4 (Figure $3 D$ ) was elevated in the NPAF scar group as determined by IHC staining when compared to the control group. The results revealed the role of S100A4 in cardiac fibrosis postNPAF catheter ablation.

\section{Circulating S100A4, atrial fibrosis, and NPAF recurrence}

A total of 93 (92.1\%) of the $101 \mathrm{AF}$ patients were followed up after the procedure, and 17 (18.3\%) had relapsed by 1-year follow-up. As shown in Table 2, there were 13 patients with AF recurrence in the NPAF scar group and 4 patients with $\mathrm{AF}$ recurrence in the non-scar group. The clinical recurrence rate was significantly higher $(27.0 \% v s$. $9.0 \%, \mathrm{P}<0.001)$ in the NPAF scar group than in the nonscar group. In addition, we found no significant differences in the laboratory indexes of BMI, SBP, DBP, TC, TG, HDL, LDL, and FBS (all $\mathrm{P}>0.05$ ), while circulating S100A4 (3.6 \pm 1.2 vs. $2.2 \pm 0.7 \mathrm{ng} / \mathrm{mL}, \mathrm{P}<0.001)$ significantly increased in the NPAF scar patients. The linear correlation between the S100A4 value and the extent of LV scar was also analyzed. There was a significant Pearson correlation between the S100A4 value and the extent of $\mathrm{LV}$ scar $(\mathrm{P}<0.001)$. This demonstrates that the extent of $\mathrm{LV}$ scar quantified by EAM) is associated with circulating levels of S100A4 in patients with NPAF following catheter ablation. Univariate predictors of NPAF clinical recurrence were investigated, including gender, age $>65$, duration of $\mathrm{AF}$ persistence, alcohol intake, smoking, BMI, SBP, DBP, TC, TG, HDL, LDL, FBS, BNP, and circulating S100A4 levels (Table 3). Among these parameters, a persistently abnormal level of S100A4 (HR: 2.87, 95\% CI: 1.99-4.14; $\mathrm{P}<0.001$ ) was the only independent predictor of adverse events. A multivariate analysis was not performed. The results suggested the relationship between NPAF recurrence and atrial fibrosis, and demonstrated the diagnostic role of S100A4 in clinical NPAF recurrence.

\section{Circulating $S 100 A 4$ predicted NPAF clinical recurrence}

Kaplan-Meier curves demonstrated that patients with NPAF and LA scars had significantly lower clinical recurrence event-free survival rates $(\mathrm{P}=0.044$, Figure $4 A)$ compared to the non-scar NPAF group. In addition, ROC curves were generated for describing the prognostic role of circulating 
A

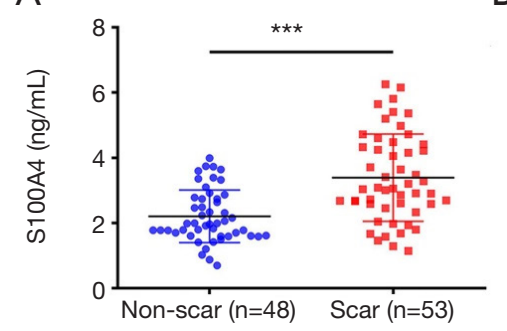

B

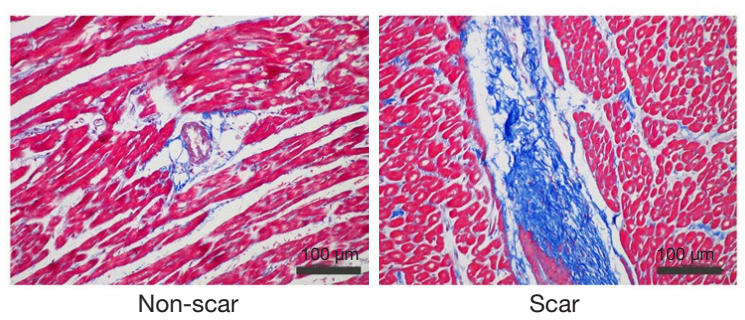

C

$B$

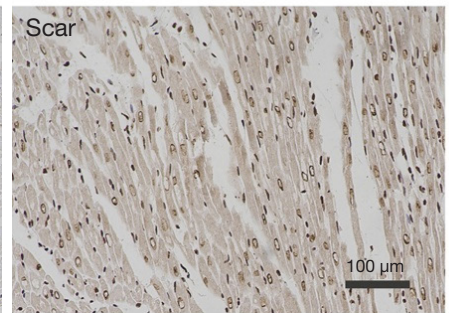

D
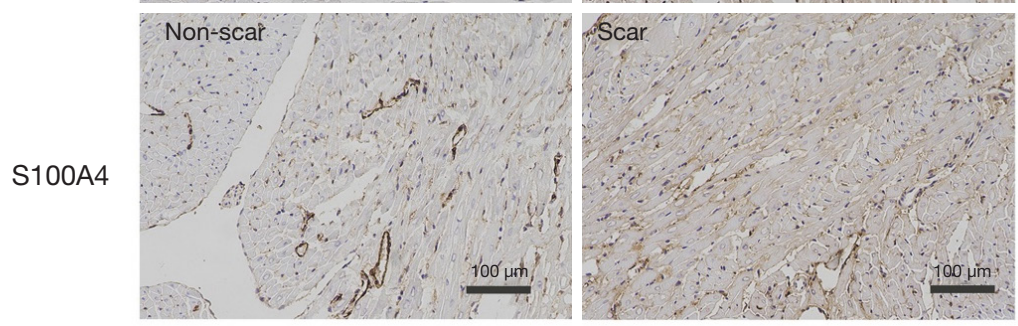

Figure 3 S100A4 characterizes left atrial scar in patients undergoing ablation for atrial fibrillation. (A) Post-ablation circulating S100A4 was elevated in the scar group compared to the non-scar group. (B-D) In the canine model, the collagen fibers around small blood vessels (B) and the levels of $\alpha$-SMA (C) and S100A4 (D) increased in the scar group as determined by Masson's trichrome staining and immunohistochemistry compared with the non-scar group. ${ }^{* * *}, \mathrm{P}<0.001$, compared with the non-scar group.

Table 2 Clinical indexes at 12-month follow-up

\begin{tabular}{|c|c|c|c|c|}
\hline Indexes & Total $(n=93)$ & Non-scar $(n=48)$ & Scar $(n=45)$ & $P$ value \\
\hline Recurrence, n (\%) & 17 (18.3) & $4(8.3)$ & $13(28.9)$ & $<0.001^{*}$ \\
\hline BMI $\left(\mathrm{kg} / \mathrm{m}^{2}\right)$ & $24 \pm 3$ & $23 \pm 3$ & $24 \pm 3$ & 0.90 \\
\hline SBP $(m m H g)$ & $126 \pm 10$ & $124 \pm 11$ & $123 \pm 9$ & 0.23 \\
\hline $\mathrm{DBP}(\mathrm{mmHg})$ & $75 \pm 7$ & $75 \pm 7$ & $74 \pm 7$ & 0.53 \\
\hline $\mathrm{TG}(\mathrm{mg} / \mathrm{dL})$ & $131 \pm 13$ & $130 \pm 14$ & $131 \pm 12$ & 0.16 \\
\hline $\mathrm{HDL}$ (mg/dL) & $39 \pm 7$ & $40 \pm 7$ & $38 \pm 7$ & 0.86 \\
\hline LDL (mg/dL) & $117 \pm 8$ & $118 \pm 8$ & $117 \pm 8$ & 0.30 \\
\hline FBS (mg/dL) & $118 \pm 19$ & $117 \pm 20$ & $119 \pm 19$ & 0.87 \\
\hline
\end{tabular}

Mean \pm SD was reported. *, $\mathrm{P}<0.05$, compared to the non-scar group. BMI, body mass index; SBP, systolic blood pressure; DBP, diastolic blood pressure; TC, total cholesterol; TG, total triglyceride; HDL, high-density lipoprotein; LDL, low-density lipoprotein; FBS, fasting blood sugar; BNP, brain natriuretic peptide; SD, standard deviation. 
Table 3 Clinical recurrence predicted by univariate analysis

\begin{tabular}{lcc}
\hline Variables & HR $(95 \% \mathrm{Cl})$ & P value \\
\hline Gender & $0.95(0.31-2.91)$ & 0.928 \\
Age >65 years & $0.99(0.35-2.82)$ & 0.988 \\
Smoking & $1.02(0.24-4.34)$ & 0.982 \\
Alcohol intake & $0.97(0.23-4.14)$ & 0.965 \\
Duration of AF & $1.00(0.97-1.03)$ & 0.854 \\
BMl & $1.10(0.93-1.30)$ & 0.267 \\
TC & $1.00(0.96-1.03)$ & 0.796 \\
TG & $0.98(0.94-1.02)$ & 0.289 \\
HDL & $1.04(0.97-1.10)$ & 0.273 \\
LDL & $0.99(0.93-1.05)$ & 0.77 \\
FBS & $1.00(0.98-1.03)$ & 0.822 \\
BNP & $1.00(1.00-1.00)$ & 0.646 \\
Scar & $2.99(0.97-9.18)$ & 0.056 \\
S100A4 & $2.87(1.99-4.14)$ & $<0.001$ \\
\hline
\end{tabular}

Mean \pm SD was reported. Univariate predictors of total events were determined by the Cox proportional hazards model. $\mathrm{HR}$, hazard ratio; $\mathrm{Cl}$, confidence interval; $\mathrm{AF}$, atrial fibrillation; TC, total cholesterol; TG, total triglyceride; HDL, high-density lipoprotein; LDL, low-density lipoprotein; FBS, fasting blood sugar; BNP, brain natriuretic peptide; SD, standard deviation.

S100A4 in NPAF clinical recurrence and stroke. The serum S100A4 model produced an AUC of 0.865 (95\% CI: 0.750 0.981 ) in predicting clinical recurrence (Figure $4 B$ ). These findings demonstrated that the serum S100A4 model had strong predictive power for NPAF recurrence post-ablation.

\section{Discussion}

The principle new findings of this study are that: (I) increased serum S100A4 level was correlated with atrial fibrosis in NPAF patients and in a canine model; (II) the recurrence rate was higher among NPAF patients with scars at long-term follow up; (III) a model of serum S100A4 level was efficient in predicting NPAF clinical recurrence.

Our study identified LA scar based on abnormal electrograms during SR mapping after circumferential PVs were isolated, CTI ablated, and SR restored. Low atrial endocardial bipolar voltage is a commonly used surrogate marker for the presence of atrial fibrosis. LVZs show obvious associations with clinical outcomes and comorbidities and serve as sites where AF is triggered. Several contemporary trials have shown the probability of using LVZs as the substrates for AF ablation (19-23). However, the accuracy of EAM voltage in defining a scar has not been validated (24). In addition, we used the EnsiteNavX system for EAM. Whether the voltage cutoff values that this system still fit other EAM systems remains uncertain (25).

Furthermore, we tested the voltage cutoffs in consecutive NPAF patients who had undergone ablation, not in patients with other types of AF or without NPAF $(6,26)$. Various noninvasive imaging techniques have been developed, such as strain imaging with a bull's-eye map (27), real-time myocardial contrast echocardiography (28), and myocardial contrast-enhanced magnetic resonance imaging (29). These techniques can provide more evidence verifying the correlation between NPAF and atrial fibrosis.

Although others have reported that remodeling processes can occur after the induction of ischemic heart disease in humans and in dogs (30), the contribution of the remodeling processes in the atrial tachyarrhythmias of this model remains unclear. In this study, atrial remodeling in the AF canine model was induced, which provides an opportunity to study the mechanisms of atrial fibrosis associated with the NPAF canine model. The natriuretic peptide system is indicative of the level of myocardial strain related to $\mathrm{AF}$, as $\mathrm{BNP}$ increases in $\mathrm{AF}$ patients (31).

A genome-wide association study (GWAS) conducted by Roselli et al. in mixed ethnicities revealed that the mutation of rs7549338, a locus within $1 \mathrm{Mb}$ downstream of S100A4, was significantly associated with AF (32). In addition, a GWAS of European ancestry by Nielsen et al. defined another single nucleotide polymorphism (SNP) $1 \mathrm{Mb}$ downstream of S100A4 (rs6689306) as an AF-related variant (33). We also searched the PhenoScanner database and found several loci, including rs146161869, rs149703806, and rs556962181, associated with AF flutter-related death, all of which have been proven to be associated with the function of S100A4 in multiple tissues (34-36). Previous studies have revealed that $\mathrm{S} 100 \mathrm{~A} 4$ regulates cardiac fibrosis after $\mathrm{MI}$ via the $\mathrm{Wnt} / \beta$-catenin (37), tumor necrosis factor $\alpha(\mathrm{TNF}-\alpha)(38)$, and transforming growth factor $\beta$ (TGF- $\beta$ ) (39) signaling pathways, but the mechanism through which S100A4 activates atrial fibrosis in NPAF remains unclear. In this study, the serum S100A4 level and clinical recurrence rate were significantly elevated in NPAF scar patients compared with those in the non-scar group, confirming the diagnostic and prognostic value of serum S100A4 in NPAF patients after ablation. However, animal models of NPAF are needed 

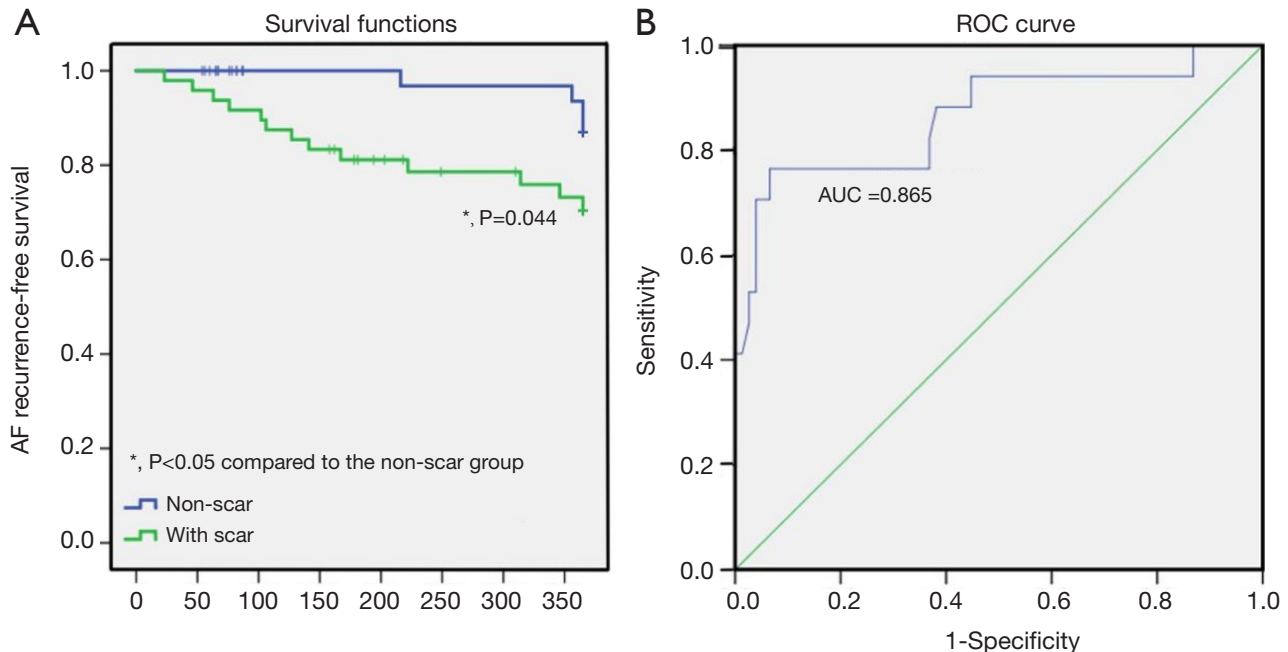

Figure 4 Predictive value of the serum S100A4 level model after successful catheter ablation in NPAF patients. (A) Kaplan-Meier curves of NPAF recurrence in the scar and non-scar groups. (B) ROC curves used for describing the specificity and sensitivity of S100A4 in characterizing the recurrence of AF. The serum S100A4 model yielded an ROC curve with an AUC of 0.865 (95\% CI: 0.750-0.981). $\mathrm{AF}$, atrial fibrillation; NPAF, non-paroxysmal atrial fibrillation; ROC, receiver operating characteristic; AUC, area under the curve; CI, confidence interval.

to verify the role of S100A4 in atrial fibrosis.

\section{Study limitations}

Based on the findings of this retrospective study, a prospectively chosen sample size of approximately 101 patients would have a power of 0.8 to detect a clinical recurrence difference of $27 \%$ in the scar group and $9 \%$ in the non-scar group. We did not add this to the current study due to its retrospective nature. Strengths include that only patients who agreed to participate voluntarily were included. It is not known to what extent the motivation of the patients might have biased the observations. Besides, in this study, echocardiographic or invasive data regarding ventricular performance during exercise were lacking. We conducted a case-control study of NPAF patients with and without scars to determine the relationship between circulating S100A4 and atrial fibrosis. The results were verified in an NPAF canine model. More evidence of circulating S100A4 in SR control and NPAF patients will be explored in the next study. Not only the presence (with LVZs) but also the extension of atrial fibrosis (at baseline, 3 months, 6 months, and 1 year post-ablation) of atrial fibrosis will be investigated in our next prospective study. More imaging, laboratory, and histological studies are needed to enhance our findings.

\section{Conclusions}

Taken together, circulating the S100A4 level is a promising marker for NPAF and can predict NPAF clinical recurrence after catheter ablation.

\section{Acknowledgments}

Funding: This study was supported by the Priority Academic Program Development of Jiangsu Higher Education Institutions (PAPD), by Jiangsu Provincial Cadre Health Care Program (Grant No.BJ17019), by the Yangzhou Key Technology R\&D Program (YZ2019067), and by the National Natural Science Foundation of China (Grant No.81871359, 82071944, and 81601516).

\section{Footnote}

Reporting Checklist: The authors have completed the STARD reporting checklist. Available at https://dx.doi. org/10.21037/atm-21-1101

Data Sharing Statement: Available at https://dx.doi. org/10.21037/atm-21-1101

Conflicts of Interest: All authors have completed the ICMJE 
uniform disclosure form (available at https://dx.doi. org/10.21037/atm-21-1101). MC serves as an unpaid editorial board member of Annals of Translational Medicine from Mar 2021 to Feb 2022. The other authors have no conflicts of interest to declare.

Ethical Statement: The authors are accountable for all aspects of the work in ensuring that questions related to the accuracy or integrity of any part of the work are appropriately investigated and resolved. The study was conducted in accordance with the Declaration of Helsinki (as revised in 2013). The clinical study was approved by the Ethics Committee of the First Affiliated Hospital of Nanjing Medical University (2018-SR-229). All procedures complied with the institutional guidelines of the First Affiliated Hospital of Nanjing Medical University Health System, and all patients signed written consent forms. The animal study was approved by the ethical committee (approval No. YFYDW2017004) and carried out according to relevant regulations of the First Affiliated Hospital of Nanjing Medical University. All animal experiments were performed following the guidelines published by the National Institutes of Health (No. 85-23, revised 1996).

Open Access Statement: This is an Open Access article distributed in accordance with the Creative Commons Attribution-NonCommercial-NoDerivs 4.0 International License (CC BY-NC-ND 4.0), which permits the noncommercial replication and distribution of the article with the strict proviso that no changes or edits are made and the original work is properly cited (including links to both the formal publication through the relevant DOI and the license). See: https://creativecommons.org/licenses/by-nc-nd/4.0/.

\section{References}

1. Savelieva I, Camm AJ. The results of pacing trials for the prevention and termination of atrial tachyarrhythmias: is there any evidence of therapeutic breakthrough? J Interv Card Electrophysiol 2003;8:103-15.

2. Wu CH, Hu YF, Chou CY, et al. Transforming growth factor- $\beta 1$ level and outcome after catheter ablation for nonparoxysmal atrial fibrillation. Heart Rhythm 2013;10:10-5.

3. Platonov PG, Mitrofanova LB, Orshanskaya V, et al. Structural abnormalities in atrial walls are associated with presence and persistency of atrial fibrillation but not with age. J Am Coll Cardiol 2011;58:2225-32.

4. Tarabykina S, Griffiths TR, Tulchinsky E, et al. Metastasis- associated protein S100A4: spotlight on its role in cell migration. Curr Cancer Drug Targets 2007;7:217-28.

5. Hordof AJ, Edie R, Malm JR, et al. Electrophysiologic properties and response to pharmacologic agents of fibers from diseased human atria. Circulation 1976;54:774-9.

6. Teh AW, Kistler PM, Lee G, et al. Electroanatomic remodeling of the left atrium in paroxysmal and persistent atrial fibrillation patients without structural heart disease. J Cardiovasc Electrophysiol 2012;23:232-8.

7. Lin Y, Yang B, Garcia FC, et al. Comparison of left atrial electrophysiologic abnormalities during sinus rhythm in patients with different type of atrial fibrillation. J Interv Card Electrophysiol 2014;39:57-67.

8. Wu Y, Valdivia HH, Wehrens XH, et al. A Single Protein Kinase A or Calmodulin Kinase II Site Does Not Control the Cardiac Pacemaker Ca2+ Clock. Circ Arrhythm Electrophysiol 2016;9:e03180.

9. Chen M. Scar homogenization in AF ablation: Evolution and practice. J Atr Fibrillation 2017;10:1618.

10. Yang $\mathrm{H}, \mathrm{Zhao} \mathrm{K}, \mathrm{Yu} \mathrm{Q}$, et al. Evaluation of plasma and tissue S100A4 protein and mRNA levels as potential markers of metastasis and prognosis in clear cell renal cell carcinoma. J Int Med Res 2012;40:475-85.

11. Xia H, Gilbertsen A, Herrera J, et al. Calcium-binding protein S100A4 confers mesenchymal progenitor cell fibrogenicity in idiopathic pulmonary fibrosis. J Clin Invest 2017;127:2586-97.

12. Taylor S, Herrington S, Prime W, et al. S100A4 (p9Ka) protein in colon carcinoma and liver metastases: association with carcinoma cells and T-lymphocytes. Br J Cancer 2002;86:409-16.

13. Le Hir M, Hegyi I, Cueni-Loffing D, et al. Characterization of renal interstitial fibroblast-specific protein 1/S100A4positive cells in healthy and inflamed rodent kidneys. Histochem Cell Biol 2005;123:335-46.

14. Schneider M, Kostin S, Strøm CC, et al. S100A4 is upregulated in injured myocardium and promotes growth and survival of cardiac myocytes. Cardiovasc Res 2007;75:40-50.

15. Qian L, Zhang Y, Zhu M, et al. Improvements in left ventricular regional and global systolic function following treatment with S100A4-shRNA after myocardial infarction in mice. Quant Imaging Med Surg 2019;9:1066-75.

16. Kato T, Sekiguchi A, Sagara K, et al. Endothelialmesenchymal transition in human atrial fibrillation. J Cardiol 2017;69:706-11.

17. Devereux RB, Roman MJ, Paranicas M, et al. Impact of diabetes on cardiac structure and function: the strong heart study. Circulation 2000;101:2271-6.

18. Yang B, Jiang C, Lin Y, et al. STABLE-SR 


\section{Page 10 of 10}

(Electrophysiological Substrate Ablation in the Left Atrium During Sinus Rhythm) for the Treatment of Nonparoxysmal Atrial Fibrillation: A Prospective, Multicenter Randomized Clinical Trial. Circ Arrhythm Electrophysiol 2017;10:e005405.

19. Boldt A, Wetzel U, Lauschke J, et al. Fibrosis in left atrial tissue of patients with atrial fibrillation with and without underlying mitral valve disease. Heart 2004;90:400-5.

20. McGann C, Akoum N, Patel A, et al. Atrial fibrillation ablation outcome is predicted by left atrial remodeling on MRI. Circ Arrhythm Electrophysiol 2014;7:23-30.

21. Spragg DD, Khurram I, Zimmerman SL, et al. Initial experience with magnetic resonance imaging of atrial scar and co-registration with electroanatomic voltage mapping during atrial fibrillation: success and limitations. Heart Rhythm 2012;9:2003-9.

22. Jadidi AS, Cochet H, Shah AJ, et al. Inverse relationship between fractionated electrograms and atrial fibrosis in persistent atrial fibrillation: combined magnetic resonance imaging and high-density mapping. J Am Coll Cardiol 2013;62:802-12.

23. Khurram IM, Beinart R, Zipunnikov V, et al. Magnetic resonance image intensity ratio, a normalized measure to enable interpatient comparability of left atrial fibrosis. Heart Rhythm 2014;11:85-92.

24. Igarashi T, Niwano S, Niwano H, et al. Linagliptin prevents atrial electrical and structural remodeling in a canine model of atrial fibrillation. Heart Vessels 2018;33:1258-65.

25. Kapa S, Desjardins B, Callans DJ, et al. Contact electroanatomic mapping derived voltage criteria for characterizing left atrial scar in patients undergoing ablation for atrial fibrillation. J Cardiovasc Electrophysiol 2014;25:1044-52.

26. Miyamoto K, Tsuchiya T, Narita S, et al. Bipolar electrogram amplitudes in the left atrium are related to local conduction velocity in patients with atrial fibrillation. Europace 2009;11:1597-605.

27. Yang LT, Kado Y, Nagata Y, et al. Strain Imaging with a Bull's-Eye Map for Detecting Significant Coronary Stenosis during Dobutamine Stress Echocardiography. J Am Soc Echocardiogr 2017;30:159-167.e1.

28. Dijkmans PA, Senior R, Becher H, et al. Myocardial contrast echocardiography evolving as a clinically feasible technique for accurate, rapid, and safe assessment of myocardial perfusion: the evidence so far. J Am Coll Cardiol 2006;48:2168-77.

29. Christiansen JP, Edwards C, Sinclair T, et al. Detection

\section{Qian et al. Prognostic value of S100A4 for atrial fibrosis in AF}

of myocardial scar by contrast-enhanced cardiac magnetic resonance imaging in patients with troponin-positive chest pain and minimal angiographic coronary artery disease. Am J Cardiol 2006;97:768-71.

30. Miyauchi Y, Zhou S, Okuyama Y, et al. Altered atrial electrical restitution and heterogeneous sympathetic hyperinnervation in hearts with chronic left ventricular myocardial infarction: implications for atrial fibrillation. Circulation 2003;108:360-6.

31. Nortamo S, Ukkola O, Lepojärvi S, et al. Association of sST2 and hs-CRP levels with new-onset atrial fibrillation in coronary artery disease. Int J Cardiol 2017;248:173-8.

32. Roselli C, Chaffin MD, Weng LC, et al. Multi-ethnic genome-wide association study for atrial fibrillation. Nat Genet 2018;50:1225-33.

33. Nielsen JB, Thorolfsdottir RB, Fritsche LG, et al. Biobankdriven genomic discovery yields new insight into atrial fibrillation biology. Nat Genet 2018;50:1234-9.

34. Kamat MA, Blackshaw JA, Young R, et al. PhenoScanner V2: an expanded tool for searching human genotype-phenotype associations. Bioinformatics 2019;35:4851-3.

35. Staley JR, Blackshaw J, Kamat MA, et al. PhenoScanner: a database of human genotype-phenotype associations. Bioinformatics 2016;32:3207-9.

36. Lu Y, Quan C, Chen H, et al. 3DSNP: a database for linking human noncoding SNPs to their three-dimensional interacting genes. Nucleic Acids Res 2017;45:D643-9.

37. Qian L, Hong J, Zhang Y, et al. Downregulation of S100A4 Alleviates Cardiac Fibrosis via Wnt/ $\beta$-Catenin Pathway in Mice. Cell Physiol Biochem 2018;46:2551-60.

38. Sato F, Kohsaka A, Takahashi K, et al. Smad3 and Bmal1 regulate p21 and S100A4 expression in myocardial stromal fibroblasts via TNF- $\alpha$. Histochem Cell Biol 2017;148:617-24.

39. Tomcik M, Palumbo-Zerr K, Zerr P, et al. S100A4 amplifies TGF- $\beta$-induced fibroblast activation in systemic sclerosis. Ann Rheum Dis 2015;74:1748-55.

(English Language Editors: C. Betlazar-Maseh and J. Chapnick)

Cite this article as: Qian L, Gong J, Ma W, Sun Y, Hong J, $\mathrm{Xu}$ D, Chu M. Circulating S100A4 as a prognostic biomarker for patients with nonparoxysmal atrial fibrillation after catheter ablation. Ann Transl Med 2021;9(18):1400. doi: 10.21037/atm21-1101 